\title{
Mössbauer and Magnetometric Studies of Spin Reorientations in $\operatorname{Er}_{2-x} \mathrm{Th}_{x} \mathrm{Fe}_{14} \mathrm{~B}$
}

\author{
A. Wojciechowska, B.F. Bogacz And A.T. Pȩdziwiatr*
}

M. Smoluchowski Institute of Physics, Jagiellonian University

Reymonta 4, 30-059 Kraków, Poland

The $\operatorname{Er}_{2-x} \operatorname{Th}_{x} \mathrm{Fe}_{14} \mathrm{~B}(x=0.5,1.0,1.5)$ polycrystalline compounds have been investigated with ${ }^{57} \mathrm{Fe}$ Mössbauer spectroscopy in the $50-330 \mathrm{~K}$ temperature range and magnetometry in the temperature range of 4.2-340 K. Initial magnetization vs. temperature measurements allowed us to establish the temperature regions of reorientations and also the Curie temperatures of the compounds. The spin reorientation phenomena (changes from planar to axial spin arrangements) have been studied extensively by a narrow step temperature Mössbauer scanning in the vicinity of the spin reorientation temperatures. From the analysis of the obtained Mössbauer spectra it was deduced that in the region of transition each subspectrum was split into two Zeeman sextets, which were characterised by different hyperfine magnetic fields and quadrupole splittings. A consistent way of describing the Mössbauer spectra in the wide range of temperatures was proposed. The composition and temperature dependences of hyperfine interaction parameters and subspectra contributions were derived from experimental spectra. The transition temperatures were determined for all the compounds studied and the spin phase diagram was constructed.

PACS numbers: $76.80 .+\mathrm{y}, 75.25 .+\mathrm{z}$

\section{Introduction}

The studies of spin reorientation phenomena in the Er-based $\mathrm{R}_{2} \mathrm{Fe}_{14} \mathrm{~B}(\mathrm{R}=$ rare earth) systems are important because they establish the temperature region of uniaxial anisotropy which is crucial for permanent magnet applications. In these compounds, the magnetocrystalline anisotropy changes from planar (basal plane) to axial (along the $c$-axis) with increasing temperature.

The easy magnetization direction of $\mathrm{Er}_{2-x} \mathrm{R}_{x} \mathrm{Fe}_{14} \mathrm{~B}$ depends on the temperature induced competition between the uniaxial Fe sublattice anisotropy [1] and the basal plane (Er,R) sublattice anisotropy [2]. Thorium is not a lanthanide, yet

*corresponding author; e-mail: ufpedziw@if.uj.edu.pl 
it was possible to incorporate it into Er sites of the crystal lattice of the $\mathrm{Nd}_{2} \mathrm{Fe}_{14} \mathrm{~B}$ type [3].

The $\mathrm{Er}_{2-x} \mathrm{Th}_{x} \mathrm{Fe}_{14} \mathrm{~B}$ crystallize in a tetragonal structure with the $P 4_{2} / m n m$ space group. Iron atoms occupy six non-equivalent crystal sites $\left(16 k_{1}, 16 k_{2}, 8 j_{1}\right.$, $8 j_{2}, 4 e, 4 c$ ), the rare earth ions occupy $4 f$ and $4 g$ crystallographic sites and boron is located at $4 g$ site [4].

In this study the polycrystalline $\mathrm{Er}_{2-x} \mathrm{Th}_{x} \mathrm{Fe}_{14} \mathrm{~B}(x=0.5,1.0,1.5)$ isostructural compounds have been investigated by ${ }^{57} \mathrm{Fe}$ Mössbauer spectroscopy and magnetometry in order to establish the spin reorientation temperatures, $T_{\mathrm{SR}}$, the influence of reorientation on hyperfine interaction parameters and to establish the influence of thorium on the transition.

\section{Experimental}

The $\operatorname{Er}_{2-x} \operatorname{Th}_{x} \mathrm{Fe}_{14} \mathrm{~B}(x=0.5,1.0,1.5)$ alloys were obtained by a standard procedure of induction melting under flowing high purity argon and subsequent annealing at $900^{\circ} \mathrm{C}$ for 2 weeks. The X-ray and thermomagnetic analyses (TMA) proved the single phase integrity of materials.

TMA was performed by recording magnetization versus temperature, $M$ vs. $T$, curves at low external magnetic field with the use of a Faraday-type magnetic balance. The Curie temperatures, $T_{\mathrm{C}}$, were also determined from those measurements.

The ${ }^{57} \mathrm{Fe}$ Mössbauer transmission spectra were recorded in the temperature range of 50-330 K. The spin reorientation phenomenon near $T_{\mathrm{SR}}$ has been studied extensively by narrow step temperature scanning using a ${ }^{57} \mathrm{Co}(\mathrm{Rh})$ source and a computer-driven constant acceleration mode spectrometer. A high purity iron foil was used to calibrate the velocity scale. Isomer shifts were established with respect to the centre of gravity of the room temperature iron Mössbauer spectrum.

\section{Data analysis}

Magnetization curves obtained at low external magnetic field for rough chunks of materials exhibited some anomalies in the vicinity of reorientations (see Fig. 1) which enabled the estimation of temperature ranges of the transitions and the approximate determination of the spin reorientation temperatures, $T_{\mathrm{SRM} 1,2}$, which were taken as inflection points of the descending portion of curve, following the procedure described in [5]. The existence of two humps on $M$ vs. $T$ curves (Fig. 1) suggests a two-stage transition.

Examples of experimental Mössbauer spectra of the $\operatorname{Er}_{0.5} \mathrm{Th}_{1.5} \mathrm{Fe}_{14} \mathrm{~B}$ alloy are shown in Fig. 2. The "exponential" approximation [6] of the transmission integral and a simultaneous fitting of several spectra was applied (as in [7]) to describe the investigated Mössbauer spectra and obtain consistent fits. For temperatures outside the transition region, the Mössbauer spectra were analysed using six Zeeman subspectra with relative intensities in agreement with iron occupation 


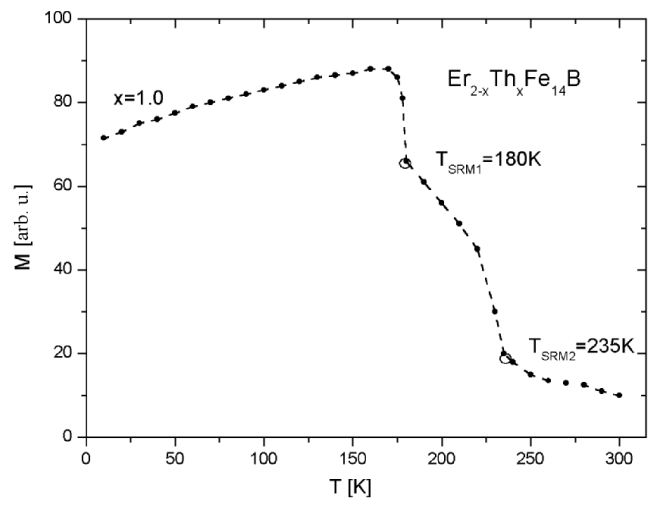

Fig. 1. Irregularities on magnetization vs. temperature curves evidenced in the vicinity of spin reorientations for $\mathrm{Er}_{1} \mathrm{Th}_{1} \mathrm{Fe}_{14} \mathrm{~B}$.

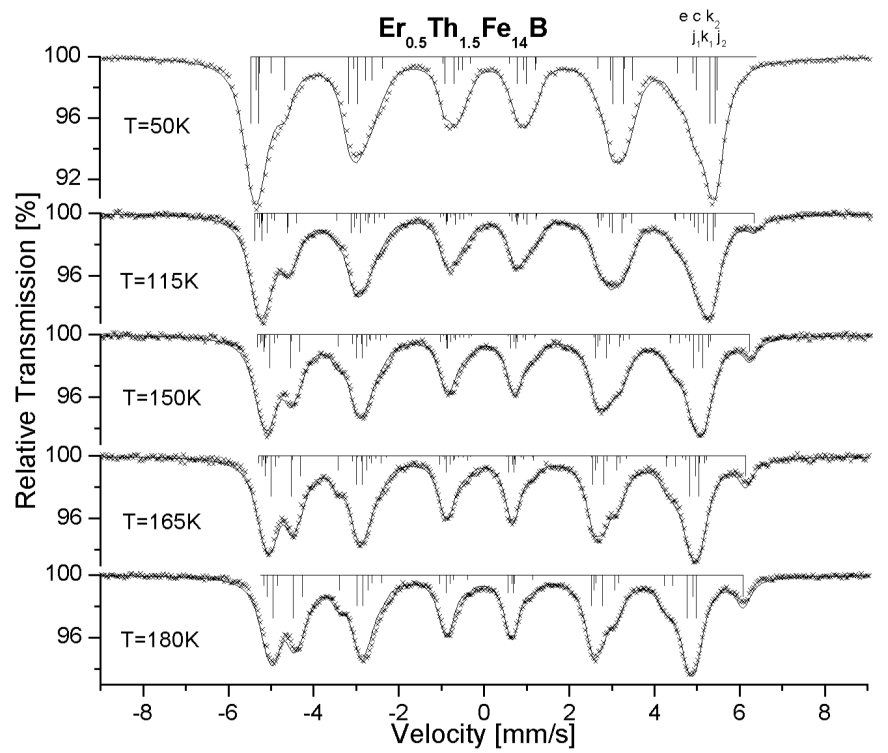

Fig. 2. The selected ${ }^{57} \mathrm{Fe}$ Mössbauer transmission spectra for the $\mathrm{Er}_{2-x} \mathrm{Th}_{x} \mathrm{Fe}_{14} \mathrm{~B}$ $(x=1.5)$ intermetallic compound. The solid lines are fits to the data. The stick diagrams show the line positions and relative intensities.

of crystallographic sublattices $(4: 4: 2: 2: 1: 1)$. For temperatures inside the region of reorientation two subspectra correspond to each sublattice.

All subspectra were characterised by the following hyperfine interaction parameters: magnetic field, $B$; isomer shift, $I S$; quadrupole splitting, $Q S$ (defined as $\left[\left(V_{6}-V_{5}\right)-\left(V_{2}-V_{1}\right)\right] / 4$, where $V_{i}$ are the velocities corresponding to Mössbauer line positions). It is significant that spectra below spin reorientation region (described by "low temperature" Zeeman sextets) and above (described by "high 
temperature" Zeeman sextets) have different values of $B$ and $Q S$. There is a coexistence of the "low" and "high temperature" Zeeman sextets in the region of reorientation. Both kinds of Zeeman sextets exchange gradually (between themselves) their contributions $C_{\mathrm{l}}, C_{\mathrm{h}}$ to the total spectrum. The weak, systematic changes of $B$ and $Q S$ with temperature were taken into account for spectra below and above spin reorientation region. A common linear temperature dependence of $I S$ caused by the second order Doppler shift effect was assumed for "low" and "high temperature" Zeeman sextets.

\section{Results and discussion}

Figures 3, 4 show the behaviour of $B$ and $Q S$ in the $\operatorname{Er}_{1} \operatorname{Th}_{1} \mathrm{Fe}_{14} \mathrm{~B}$ compound. It is visible that hyperfine field decreases with the increase in temperature, whereas for the quadrupole splitting the temperature dependence is very weak. Moreover,

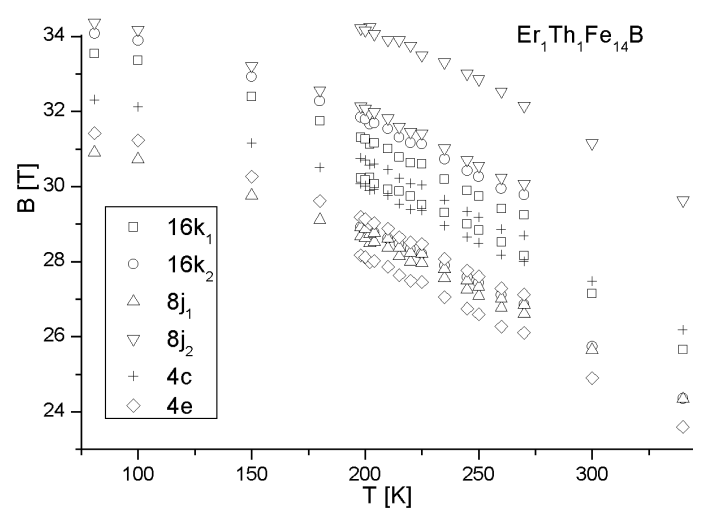

Fig. 3. Temperature dependence of the hyperfine magnetic field, $B$, for different crystal sites of the $\mathrm{Er}_{1} \mathrm{Th}_{1} \mathrm{Fe}_{14} \mathrm{~B}$ compound. The average error is $0.1 \mathrm{~T}$.

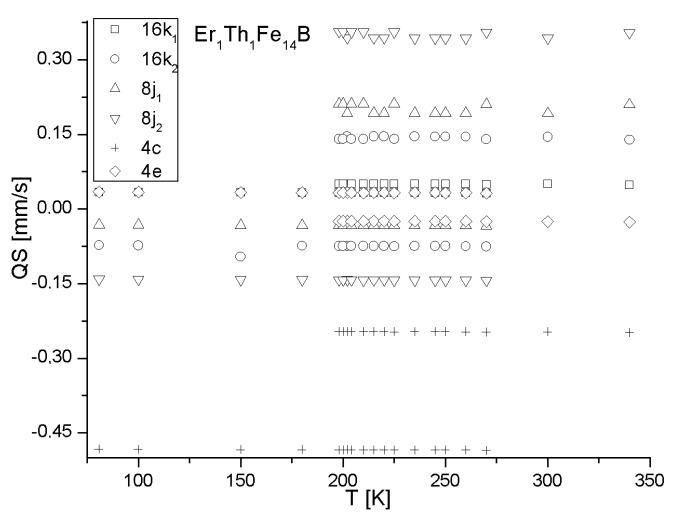

Fig. 4. Temperature dependence of the quadrupole splitting, $Q S$, for different crystal sites of the $\mathrm{Er}_{1} \mathrm{Th}_{1} \mathrm{Fe}_{14} \mathrm{~B}$ compound. The average error is $0.01 \mathrm{~mm} / \mathrm{s}$. 
in the region of reorientation each subspectrum is characterised by two Zeeman sextets with different $B$ and $Q S$.

The spin reorientation phenomenon seems to be a two-stage process for $\mathrm{Er}_{2-x} \mathrm{Th}_{x} \mathrm{Fe}_{14} \mathrm{~B}(x=1.0,1.5)$ compounds, as indicated by the two humps on $M$ vs. $T$ curves and confirmed by Mössbauer studies. The contributions $C_{\mathrm{l}}, C_{\mathrm{h}}$ of

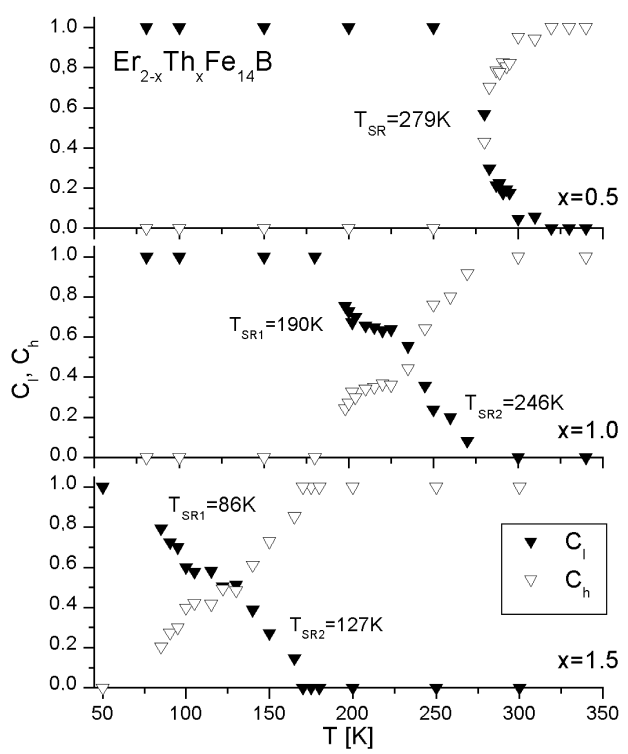

Fig. 5. Temperature dependences of subspectra contributions for both $C_{1}$ — "low temperature" (solid triangle) and $C_{\mathrm{h}}$ — "high temperature" (open triangle) Zeeman sextets for $\mathrm{Er}_{2-x} \mathrm{Th}_{x} \mathrm{Fe}_{14} \mathrm{~B}$. The average error of $T_{\mathrm{SR}}$ is $5 \mathrm{~K}$.

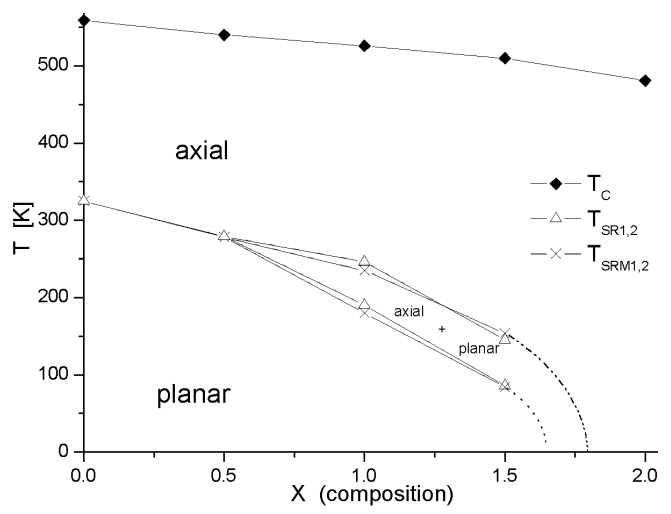

Fig. 6. Spin structure phase diagram for the $\mathrm{Er}_{2-x} \mathrm{Th}_{x} \mathrm{Fe}_{14} \mathrm{~B}$ compounds. $T_{\mathrm{C}}-$ Curie temperature, $T_{\mathrm{SR} 1,2}$ - spin reorientation temperatures determined from Mössbauer measurements, $T_{\mathrm{SRM} 1,2}$ - spin reorientation temperatures determined from magnetic measurements. Dotted line - hypotetical line to guide the eye (the theoretical calculations for similar compounds [8] indicate that the lines should have a curvature). 
both "low" and "high temperature" Zeeman sextets for $\operatorname{Er}_{2-x} \mathrm{Th}_{x} \mathrm{Fe}_{14} \mathrm{~B}(x=1.0$, 1.5) compounds (shown in Fig. 5) also show a two-stage character. From this plot the $T_{\mathrm{SR} 1,2}$ (the temperature corresponding to reorientation of half the number of spins on a given stage of reorientation) were derived. It was found that the substitution of Th for Er causes the decrease in spin reorientation temperature and the reduction of planar anisotropy range.

The spin phase diagram, which summarises the results of this study, is shown in Fig. 6. The two-stage process is visible for compounds with $x>0.5$.

\section{References}

[1] G. Givord, H.S. Li, R. Perrier de la Bathie, Solid State Commun. 51, 857 (1984).

[2] S. Hirosawa, Y. Matsuura, H. Yamamoto, S. Fujimura, M. Sagawa, H. Yamauchi, J. Appl. Phys. 59, 873 (1985).

[3] K.H.J. Buschow, H.M. Van Noort, D.B. De Mooij, J. Less-Common Met. 109, 79 (1985).

[4] J.F. Herbst, J.J. Croat, F.E. Pinkerton, W.B. Yelon, Phys. Rev. B 29, 4176 (1984).

[5] E.B. Boltich, A.T. Pȩdziwiatr, W.E. Wallace, J. Magn. Magn. Mater. 66, 317 (1987).

[6] B.F. Bogacz, Molec. Phys. Rep. 30, 15 (2000).

[7] R. Wielgosz, A.T. Pȩdziwiatr, B.F. Bogacz, S. Wróbel, Molec. Phys. Rep. 30, 167 (2000).

[8] A.T. Pȩdziwiatr, B.F. Bogacz, R. Gargula, Nukleonika 48, (Suplement 1), 59 (2003). 